\section{A sensitivity comparison of the Quick and Owren prothrombin time methods in oral anticoagulant therapy}

\author{
Juha Horsti \\ Department of Clinical Chemistry, Centre \\ for Laboratory Medicine, Tampere \\ University Hospital, Tampere, Finland
}

\section{Abstract}

Prothrombin time (PT) is the leading test for monitoring oral anticoagulation therapy (OAT). According to the World Health Organization recommendation, International Normalized Ratio (INR) results obtained from the same patient samples with the major PT methods (Quick and Owren) should be the same when the therapeutic range is the same. In our study blood samples were obtained from 207 OAT patients. We analyzed the samples using two Quick and two Owren PT (combined thromboplastin) reagents for INR and assessed the sensitivity and true coagulation activity using a new-generation PT method. The INR values with the Quick PT and Owren PT methods were very similar around the normal range, while unacceptable differences were seen within the therapeutic range and at higher INR values. The Quick PT results as INR are clearly lower than those given by Owren PT and the difference increases toward higher INR. The new PT method functions well with both Owren PT reagents, and we can calculate the true active INR. The Quick PT methods show no sensitivity to coagulation inhibition measurement. The harmonization of the INR is an important goal for the safety of OAT patients. More accurate INR results reduce morbidity and mortality, and the therapeutic ranges should be similar worldwide. In this study we found unacceptable differences in INR results produced by the two PT methods. The new method showed a lack of sensitivity to Quick PT. For the global harmonization of OAT therapy and for INR accuracy only the more sensitive 0wren PT method should be used.

\section{Introduction}

Oral anticoagulation therapy (OAT) is one of the most commonly used medications worldwide. The purpose of the treatment is to balance the risks of hemorrhage and thrombosis in the patient. Arterial and venous complications commonly are involved in morbidity and mortality in OAT patients globally. ${ }^{1-4}$ The vita- min $\mathrm{K}$ antagonists coumarin and warfarin are inexpensive and the most widely used medicines in the prevention and treatment of thromboembolism in various clinical situations, and the benefit of OAT has been proved. ${ }^{5-12}$ The major drawback with warfarin is a narrow therapeutic window and individually variable responses to the treatment. Thus frequent prothrombin time (PT) checks are required to ensure that anticoagulation remains within the therapeutic range, which is 2.0-3.0 International Normalized Ratio (INR).

New medications for oral anticoagulation therapy have been developed over a number of years and anticipated without laboratory test control in an effort to replace warfarin. The new medicines, however, have not proved superior to warfarin. The new molecules are expensive and involve serious side effects or only narrow indications for $0 \mathrm{AT} .^{13}$

According to a recent review, ${ }^{14,15}$ prothrombin time has served as a basis for OAT monitoring since its first description by Quick more than 70 years ago. PT measures vitamin Kdependent coagulation factors II, VII, and X. ${ }^{3.4}$ Warfarin (VKAs) inhibits the synthesis of coagulation factors II, VII, IX, and X in the liver, which remain partially inactive unless nine to 13 of the amino-terminal glutamate (Glu) residues are carboxylated to form $\mathrm{Ca}^{2+}$-binding $\gamma$-carboxyglutamate (Gla) residues., ${ }^{3,4}$ Thus it is inevitable that the more medication is administered, the more inactive coagulation factors will result, hampering and inhibiting the PT measurement.

Today, two major PT methods are accepted for anticoagulant medication control and they are used globally for chronic anticoagulation checks: either the Quick PT, which is based on the technique described by Quick and his colleagues in $1935,{ }^{14,15}$ or the Owren $\mathrm{PT}^{16}$ (combined thromboplastin reagent). The Owren PT is the predominant approach used in the Nordic countries, the Benelux, and Japan. The accuracy and comparability of PT results are essential to the safety of the individual in anticoagulant therapy and to improve the applicability of anticoagulation guidelines. The World Health Organization (WHO) recommendation for the use of INR given in the mid-1980s was aimed to harmonize PT results for OAT, regardless of the laboratory, reagent, instrument, or method used. ${ }^{17,18}$

Unfortunately, an increasing body of evidence indicates that this goal has not been achieved. ${ }^{1926}$

Different reagents are known to vary with respect to the source of thromboplastins and other reagent components, ${ }^{26}$ and these have not been characterized in detail. The Owren method is reagarded as the "reference method" in which fibrinogen and factor $\mathrm{V}$ are added to the reagent, and these do not depend on K-vitamin antagonists. The Quick PT meas-
Correspondence: Juha Horsti, Tampere University Hospital, Centre for Laboratory Medicine, P.0. Box 2000, FIN-33521 Tampere, Finland. E-mail: juha.horsti@pshp.fi

Key words: International Normalized Ratio, oral anticoagulant therapy, prothrombin time, warfarin.

Acknowledgments: the author thanks Kari Borchers and Henrik Biström from ILS Scandinavia for their cooperation and invaluable help.

Received for publication: 23 June 2009.

Revision received: 3 September 2009.

Accepted for publication: 3 September 2009.

This work is licensed under a Creative Commons Attribution 3.0 License (by-nc 3.0).

\section{() Copyright J. Horsti 2009}

Licensee PAGEPress, Italy

Hematology Reviews 2009; 1:e15

doi:10.4081/hr.2009.e15

ures both of these coagulation factors, which is a drawback in OAT control. In the Owren method the sample volume in the reaction mixture is 5\% only, in the Quick method 33\%. On account of this difference in sample dilution the Owren PT is considered to be a more sensitive method than the Quick PT.

In the present study, we sought to compare INR results for warfarin therapy from the same OAT patient samples using both the 0wren and the Quick PT methods. The aim was to study the method and reagent sensitivity of Owren and Quick PT using the new-generation PT method developed by Horsti et al., which can measure active coagulation without inhibition from inactive coagulation factors. ${ }^{13,27,28}$

\section{Materials and Methods}

\section{Patients and blood sampling}

Venous blood samples were obtained from 207 hospital and health-center patients referred to the PT test for the monitoring of oral anticoagulant therapy. In our region a "PINR" test code is used for this purpose. Hence, the patient samples represented all possible phases of anticoagulation: pretreatment, doseadjusting phase, and steady-state phase. All the procedures were approved by the responsible committee of our institution in accordance with the Helsinki Declaration of 1975. The blood (3.15 mL) was drawn into citrate coagulation tubes (Greiner Labortechnik $\mathrm{GmbH}$, Vacuette cat. No. 454332, 9NC) containing 0.35 $\mathrm{mL} 0.109 \mathrm{~mol} / \mathrm{L}(3.2 \%)$ citrate solution. The sample needle (Terumo, Venoject needle, Quick Fit, cat. No. MN-2138MQ) was $0.8 \times 40$ 
$\mathrm{mm}$. The sample tubes were centrifuged at $1850 \mathrm{~g}$ for $10 \mathrm{~min}$ at $20^{\circ} \mathrm{C}$ to separate the plasma. All the measurements were commenced within eight hours from blood collection.

\section{Prothrombin time determination}

The calculation formula is for the International Normalized Ratio: INR = (sam-

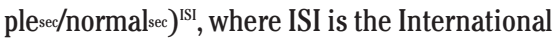
Sensitivity Index. When ISI is close to 1.0, the reagent is sensitive and ISI is insignificant in the INR calculation. The PT coagulation times were measured using a fully automated ACL TOP CTS coagulation analyzer (Instrumentation Laboratory, IL, Lexington, MA, USA). For the Owren PT (combined thromboplastin reagent) the coagulation reaction contained $10 \mu \mathrm{L}$ of citrated sample plasma, $60 \mu \mathrm{L}$ of diluent, and $140 \mu \mathrm{L}$ of reagent. The volumes for "dilution measurement" were $5 \mu \mathrm{L}+65 \mu \mathrm{L}+$ $140 \mu \mathrm{L}$. The test reagents were Nycotest PT, cat. No. 1002488 (rabbit brain thromboplastin) and diluent (Nycotest PT, dilution liquid, cat.No. 999002) from Axis-Shield as lot.10132661, ISI=1.12; Owren's PT, cat.no. GHI 131-10 (rabbit thromboplastin), and diluent (Owren's buffer, cat.No. GHI 150) from Medirox as lot 75051, ISI=1.16. For the onestage prothrombin time with Quick, $100 \mu \mathrm{L}$ of coagulation reagent was added to $50 \mu \mathrm{L}$ of citrated plasma. Sample volumes for the dilution were $100 \mu \mathrm{L}+25 \mu \mathrm{L}+25 \mu \mathrm{L}$ (Factor Diluent, HemosIL, cat.no. 0009757600 from IL). The test reagents were: RecombiPlasTin $2 \mathrm{G}$ cat. No. 0020003050 (recombinant human tissue thromboplastin) from Instrumentation Laboratory, Lexington USA as lot. N0486316, ISI=0.97; PT-Fibrinogen HS PLUS cat. no. 0008469810 (rabbit brain thromboplastin) from Instrumentation Laboratory, Lexington USA as lot. N0185573 ISI=1.11.

\section{Additional reagents}

Control Plasma Normal cat.No. 1002387 from Axis-Shield as lot. 1D71AOA, NKP Normal Control Plasma cat.No. GHI 162 from MediRox $\mathrm{AB}$ as lot. 72011, and Normal Control Assayed cat.No. 0020003110 from IL as lot. N0386069.

\section{The new-generation prothrombin time method}

We constructed PT (sec) vs. C (C = plasma or calibrator dilution factor) plots for normal and OAT plasmas. This is consistent with an uncompetitive inhibition principle with oral anticoagulants. From the line equation the $y$ axis intercept is calculated. This is the socalled minimal clotting time ( $\left.t_{\min }\right)$ with an infinite number of clotting factors. The difference in intercepts ( $y$-axis) between normal plasma and OAT plasma indicates the action of uncompetitive inhibition in seconds without a calibration effect. We went on to calculate the dif-

Table 1. Differences in INR results between four PT methods at variable INR levels as INR units and percentages. The correlation equations between methods were used for the calculations.

\begin{tabular}{lccccccccc}
$\begin{array}{l}\text { Nycotest } \\
\text { PT INR }\end{array}$ & $\begin{array}{c}\text { Owren's } \\
\text { PT INR }\end{array}$ & $\begin{array}{c}\text { Diff } \\
\text { P }\end{array}$ & $\begin{array}{c}\text { Diff } \\
\text { INR }\end{array}$ & $\begin{array}{c}\text { PT-Fib } \\
\text { INR }\end{array}$ & $\begin{array}{c}\text { Diff } \\
\%\end{array}$ & $\begin{array}{c}\text { Diff } \\
\text { INR }\end{array}$ & $\begin{array}{c}\text { Reco } \\
2 \text { G INR }\end{array}$ & $\begin{array}{c}\text { Diff } \\
\%\end{array}$ & $\begin{array}{c}\text { Diff } \\
\text { INR }\end{array}$ \\
1,00 & 1,00 & 0,05 & 0,00 & 1,08 & 8,03 & $\begin{array}{c}- \\
0,08\end{array}$ & 1,01 & 0,74 & - \\
2,00 & 1,90 & 5,25 & 0,11 & 1,88 & 6,23 & 0,12 & 1,68 & 16,16 & 0,32 \\
\hline 3,00 & 2,79 & 6,98 & 0,21 & 2,67 & 10,99 & 0,33 & 2,35 & 21,79 & 0,65 \\
4,00 & 3,69 & 7,85 & 0,31 & 3,47 & 13,37 & 0,53 & 3,02 & 24,61 & 0,98 \\
\hline 5,00 & 4,58 & 8,37 & 0,42 & 4,26 & 14,79 & 0,74 & 3,69 & 26,30 & 1,32 \\
6,00 & 5,48 & 8,72 & 0,52 & 5,06 & 15,75 & 0,94 & 4,35 & 27,43 & 1,65 \\
\hline
\end{tabular}

ference in intercepts also in INR units and subtracted this from total INRTot (more explanation about the new method in reference 28 [patent pending for PT method]), ${ }^{27,28}$

$\mathrm{INR}_{\text {Act }}=\mathrm{INR}^{\mathrm{Tot}}-\mathrm{INR}_{\text {Inh }}$

INRs were calculated using the formula INR $=$ (samplesec / normalsec) $^{\mathrm{ISI}}$

The dilution factor for the Owren PT and Quick PT was 2.0.

\section{Analytical imprecision and statistics}

The within-run precision of the PT tests was measured using normal plasma ( $\mathrm{n}=10$ determinations). The respective CVs were: for Nycotest PT 1.81\%; Owren's PT 0.90\%; RecombiPlasTin 2 G 0.55\%; PT-Fibrinogen HS PLUS 2.15\%, and pooled plasma (about 2 INR): for Nycotest PT 1.26\%; Owren's PT 1.14\%; RecombiPlasTin 2 G 1.07\%; PT-Fibrinogen HS PLUS 1.94\%. The Microsoft Excel 5.0 and Analyse-it for Microsoft Excel from Analyse-it Software Ltd programs were used to obtain the correlation functions and INR results.

\section{Results}

We compared the results of four different commercial INR determination methods (two Quick PT and two Owren PT, ISI values 0.97; 1.11 and 1.12 ; 1.16) from 207 blood samples from patients in imminent or ongoing OAT. All the ISI values used for reagents were manufacturer values. The INR values obtained with the different methods were similar around the normal range or INR 1 (Figure 1). In contrast, marked differences were seen in the therapeutic range (2-3 INR) and higher INR values between the Quick PT and Owren PT methods. The INRs produced by the Quick PT are clearly lower than those given by the Owren PT, and the difference increases toward higher INRs. Using correlation equations between the four methods, the differences were calculated applying the Microsoft Excel 5.0 and Passing \& Bablok method comparison, Analyse-it for
Microsoft Excel from Analyse-it Software Ltd programs (Table 1).

The difference between the 0wren PT methods in INR terms is below $10 \%$ over the whole measuring range. In the therapeutic range, the average difference is 0.16 INR; $6.10 \%$. The difference between Nycotest PT and Pt-Fib HS is $8.03-15.75 \%$ and in the therapeutic range an average of 0.23 INR; $8.6 \%$. The difference between Nycotest PT and Recomb 2G is 0.74$27.43 \%$ and in the therapeutic range an average of 0.49 INR; $19.0 \%$.

The Owren methods showed excellent correlation $(\mathrm{y}=0.96 \mathrm{x}+0.052$; intercept $95 \% \mathrm{CL}$ 0.034 to 0.071 ; slope 0.948 to 0.973 ), while the correlations between Quick and Owren PT were not good: Nyco PT vs. Pt-FibHS (y = $0.7955 \mathrm{x}+0.2802$; intercept $95 \%$ CL 0.242 to 0.319 ; slope 0.769 to 0.823 ), and Nyco PT vs. $\operatorname{Rec} 2 \mathrm{G}(\mathrm{y}=0.669 \mathrm{x}+0.3357$; intercept 95\% CL 0.290 to 0.381 ; slope 0.642 to 0.700 ). The correlation was not good either between the Quick methods: Pt-FibHS vs. Rec 2G (y = 0.8267x + 0.1055 , non-linear relationship; intercept $95 \%$ CL 0.063 to 0.150 ; slope 0.800 to 0.857 ).

The new-generation PT method functions well with the two Owren PT methods and makes it possible to calculate the true active INR without inhibition (Figure 2). The correction lowers the INR values closer to Quick PT values. Marked individual differences were seen in the therapeutic range 2-3 INR and higher values between Rec 2 G INR (Quick PT) and Owren's PT INRACT (Owren PT) (Figure 3 ). The correlation between active INR results is good $(\mathrm{y}=0.8955 \mathrm{x}+0.104$; intercept $95 \%$ CL 0.087 to 0.122 ; slope 0.885 to 0.907 ). The Quick PT methods show no sensitivity to the coagulation inhibition measurement.

\section{Discussion}

The global harmonization of INR and therapeutic ranges for different clinical indications is an important goal for the benefit of patients 

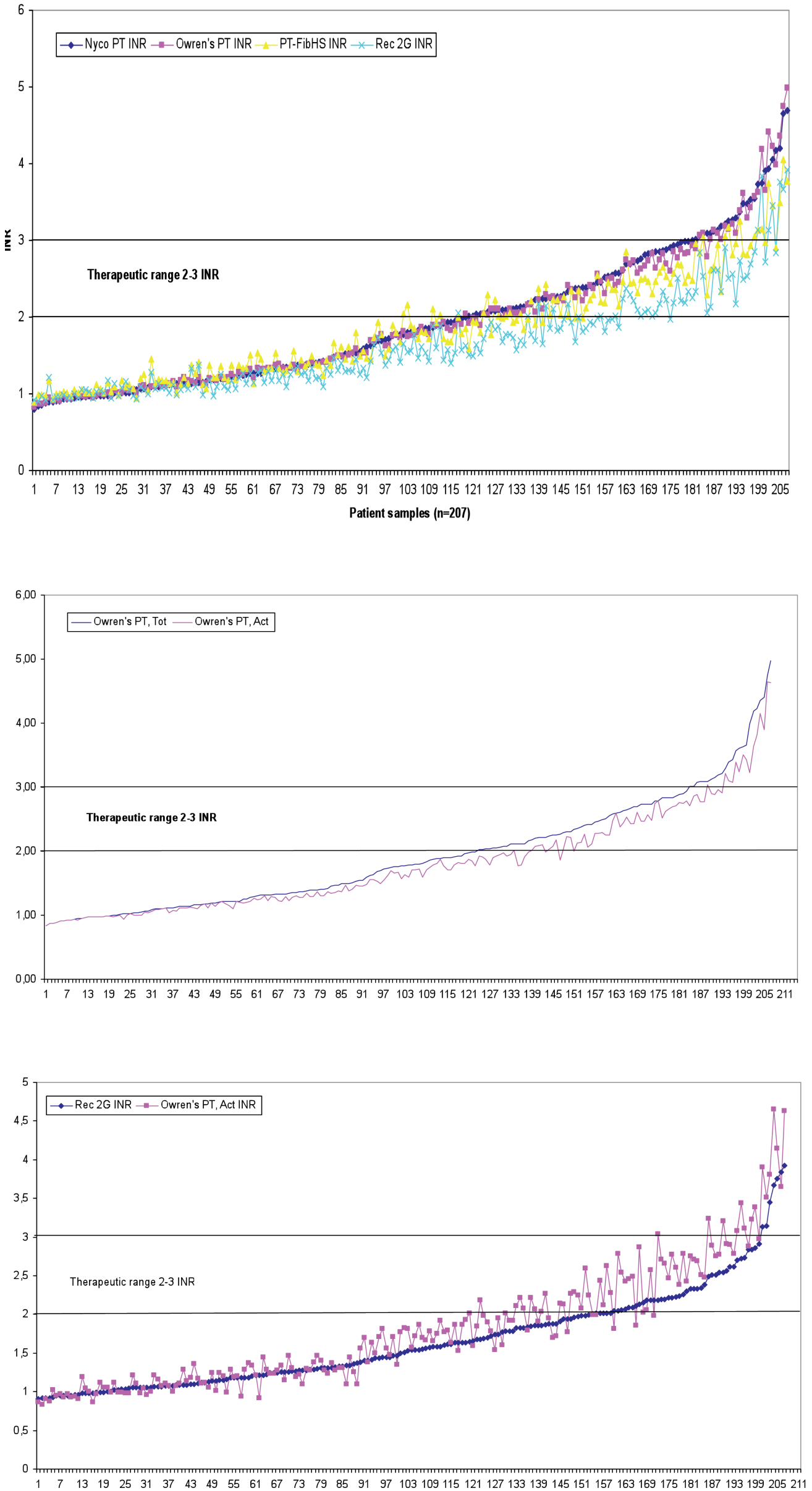

Figure 1. INR values in increasing order from 207 analyzed patient samples on anticoagulation therapy. Nycotest PT and Owren's PT are Owren methods (combined thromboplastin reagent), and $P T$ FibHS and Rec 2G are Quick methods.

Figure 2. INRтот and INRAC values in increasing order from 207 analyzed patient samples on anticoagulation therapy. Owren's PT reagent (combined thromboplastin reagent) and the new-generation PT method are used for INR measurement.

Figure 3. Rec 2G, Quick PT, INR, and Owren's PT (combined thromboplastin reagent) INRACT values in increasing order from 207 analyzed patient samples on anticoagulation therapy. The new-generation PT method is used for INRACT measurement. 
as well as clinicians. WHO originally envisaged INR as a reliable and safe method for obtaining results from anticoagulation trials and as a basis for expert guidelines for treatment. However, the results of earlier studies and this present study demonstrate that the goal has not been achieved. The narrow therapeutic range and the increase in complications and mortality outside this range call for the development of more accurate laboratory analytics. A computer simulation study of serial INR measurements has been conducted within the most widely used therapeutic range (INR 2.0 3.0 ); the authors concluded that analytical imprecision should be $<5 \%$ and analytical bias $< \pm 0.2$ INR units. ${ }^{29}$

In this study, agreement between the Quick PT and Owren PT methods was particularly poor and failed to meet the qualification requirements. For this study we selected a newly developed recombinant PT reagent, but the result was not acceptable. We may wonder whether the difference in sample volume in the reaction mixture (5\% and $33 \%$ ) accounts for the measurement sensitivity. Both Owren PT reagents are sensitive to inactive coagulation factor measurement (inhibition), while the Quick PT reagents are not.

In our earlier study we used DadeBehring Innovin with human placenta thromboplastin (Quick PT reagent), which is the most sensitive reagent to inactive coagulation factors and inhibition. The sensitivity thus does not depend only on differences in principle between the Quick PT or Owren PT methods. The thromboplastin and other components used in the reagent affect the reagent sensitivity. Horsti ${ }^{24}$ has compared the Quick PT and Owren PT methods for the harmonization of INR results and concluded that Quick PT yields clinically divergent and Owren PT clinically acceptable INR results. It would be interesting to study the effect of lupus anticoagulants on Quick PT and 0wren PT methods. The results of recent studies, unfortunately, demonstrate that attempts at harmonization have to improve. ${ }^{25}$ The unacceptable situation for oral anticoagulation was confirmed further by this present investigation.

How can the harmonization of INR results be improved worldwide? For global use, WHO should recommend only the Owren PT, which is the superior method. The advantages of Owren PT are the fact that it measures only vitamin K-dependent coagulation factors F II, F VII, and F X (not fibrinogen and F V), and provides greater sensitivity and extensive dilution of the interfering matrix substances in the final reaction mixture. ${ }^{19,25}$ The patient samples also contain inactive coagulation factors, the amount of which increases concomitant with increased anticoagulant medication and higher INR levels, interfering with and causing error in the coagulation measurement. The inhibition depends on the medication and the patient's individual metabolism, and thus each patient's sample must be corrected individually. ${ }^{27,28}$ The Owren PT methods are sensitive, but more susceptible to the inactive coagulation factors and inhibition in measurement. In an earlier study we measured only the active coagulation factors, INRAct (F II, F VII, FX) without inhibition, which provided a new possibility to develop anticoagulant therapy and more appropriate care for OAT patients. The active coagulation factors are responsible for thrombosis or bleeding in vivo and medication should be based on this principle. ${ }^{13,27,28}$ The inhibition interferes with the calibration procedure and measurement.

This study was conducted from the point of view of a clinical laboratory without clinical data and outcomes are missing between methods (Quick PT, Owren PTтот, Owren PTAст), which would give the final answer as to the superiority of PT methods. This study revealed the lack of sensitivity in both Quick PT methods but good sensitivity and correlation in the Owren PT activity (INRAct) measurements. The editorials of Clinical Chemistry have posed the critical question and sought answers: "Has the Time Arrived to Replace the Quick Prothrombin Time Test for Monitoring Oral Anticoagulant Therapy?" 19 On the basis of recent studies and our opinion we can answer: "Yes, with the Owren PT without inhibition."

\section{References}

1. Palareti G, Leali N, Coccheri S, et al. Bleeding complications of oral anticoagulant treatment: an inception cohort, prospective collaborative study (ISCOAT). Lancet 1996;348:423-8.

2. Odén A, Fahlén M. Oral anticoagulation and risk of death: a medical record linkage study. BMJ 2002;325:1073-5.

3. Ansell J, Hirsh J, Poller L, et al. The pharmacology and management of the vitamin $\mathrm{K}$ antagonists: the seventh ACCP Conference on Antithrombotic and Thrombolytic Therapy. Chest 2004;126: 204-33.

4. Hirsh J, Fuster V, Ansell J, et al. American Heart Association/American College of Cardiology Foundation Guide to Warfarin Therapy. Circulatione 2003;107:1692-734.

5. Turpie AG. Safer anticoagulant therapy after heart valve replacement. Recommendations for less intense regimens. Postgrad Med 1997;101:85-6, 89-90, 93-4.

6. Hirsh J. Optimal intensity and monitoring warfarin. Am J Cardiol 1995;75:39-42.

7. Stein PD, Alpert JS, Bussey HI, et al. Antithrombotic therapy in patients with mechanical and biological prosthetic heart valves. Chest 2001;119:220-7.
8. Hirsh J, Dalen JE, Anderson DR, et al. Oral anticoagulants' mechanism of action, clinical effectiveness, and optimal therapeutic range. Chest 1998;114:445-69.

9. Schulman S. Care of patients receiving long-term anticoagulant therapy. N Engl J Med 2003;349:675-83.

10. Gage BF, Fihn SD, White RH. Management and dosing of warfarin therapy. Am J Med 2000;109:481-8.

11. Wilson SE, Watson HG, Crowther MA. Low dose oral vitamin $\mathrm{K}$ for management of asymptomatic patients with an elevated INR: a brief review. CMAJ 2004;170:821-4.

12. Dunn AS, Turpie AG. Perioperative management of patients receiving oral anticoagulants. Arch Int Med 2003;163:901-8.

13. Horsti J. An improved method for PT measurement in OAT patients. Clin Lab Int 2008;32:16-8.

14. Quick AJ, Stanley-Brown M, Bancroft FW. A study of the coagulation defect in hemophilia and in jaundice. Am J Med Sci 1935; 190:501-11.

15. Quick AJ. The prothrombin time in haemophilia and in obstructive jaundice. J Biol Chem 1935;109:73-4.

16. Owren PA. Thrombotest. A new method for controlling anticoagulant therapy. Lancet 1959;2:754-8.

17. WHO Expert Committee on Biological Standardisation. Thirty-third Report. Technical Report Series 687. WHO Geneva 1983:81-105.

18. International Committee for Standardisation in Haematology. International Committee on Thrombosis and Haemostastis. ICSH/ICTH recommendations for reporting prothrombin time in oral anticoagulant control. Thromb Haemost 1985; 53:155-6.

19. Jackson CM, Esnouf MP. Has the time arrived to replace the quick prothrombin time test for monitoring oral anticoagulant yherapy? Clin Chem 2005;51:483-5.

20. Cunningham MT, Johnson GF, Pennell BJ, et al. The reliability of manufacturer-determined, instrument-specific international sensitivity index values for calculating the international normalised ratio. Am J Clin Pathol 1994;102:128-33.

21. van Rijn JL, Schmidt NA, Rutten WP. Correction of instrument- and reagentbased differences in determination of the International Normalised Ratio (INR) for monitoring anticoagulant therapy. Clin Chem 1989; 5:840-3.

22. Ng VL, Levin J, Corash L, et al. Failure of the International Normalised Ratio to generate consistent results within a local medical community. Am J Clin Pathol 1993;99:689-94.

23. Horsti J. Agreement of owren and quick prothrombin times: effects of citrate and 
calcium concentrations and international sensitivity index correction. Clin Chem 2001;47:940-3.

24. Horsti J. Comparison of quick and owren prothrombin time with regard to the harmonisation of the international normalised ration (INR) system. Clin Chem Lab Med 2002;40:399-403.

25. Horsti J, Uppa H, Vilpo J. Poor agreement between different prothrombin time International Normalized Ratio (INR) methods: comparison of seven commercial reagents. Clin Chem 2005;51:553-60.

26. van den Besselaar AM. International standardisation of laboratory control of oral anticoagulant therapy: a survey of thromboplastin reagents used for prothrombin time testing. J Heart Valve Dis 1993;2:4252.

27. Horsti J, Uppa H, Vilpo JA. A New generation prothrombin time method for INR. The Open Medicinal Chem J 2008;2:11-15.
28. Horsti J, Uppa H, Vilpo JA. A new generation method for quick and owren PT. The Open Hematol J 2008;2:81-5.

29. Kjeldsen J, Lassen JF, Petersen PH, et al. Biological variation of International Normalised Ratio for prothrombin times, and consequences in monitoring oral anticoagulant therapy: computer simulation of serial measurements with goal-setting for analytical quality. Clin Chem 1997;43:2175-82. 